\title{
HELMHOLTZ PMUT: DEMONSTRATING PASSIVE AMPLIFICATION IN MICROFABRICATED ACOUSTIC TRANSDUCERS
}

\author{
Anton A. Shkel and Eun Sok Kim
}

\section{Department of Electrical Engineering, University of Southern California, Los Angeles, California, USA}

\section{ABSTRACT}

This paper reports the modelling, parametric design, fabrication, and measurement of a piezoelectric micromachined ultrasonic transducer (PMUT) exploiting the Helmholtz acoustic resonance effect to acoustically amplify the electrical response. An analytical model based on electroacoustics was developed and verified by acoustic FEM simulation. The model was applied to generate a set of design parameters, and a Helmholtz PMUT was fabricated and characterized. The device's acoustic response was measured showing an improvement in sensitivity by a factor of 2.16 , demonstrating the merits of this approach.

\section{INTRODUCTION}

Acoustic transducers with high sensitivity are favored in a variety of applications including ranging, ultrasonic imaging, and acoustic signature detection in noisy environments [1]. This paper demonstrates coupling of a mechanical resonator to a resonant air cavity. Such a geometry employs the Helmholtz resonance effect, as a passive mechanism for amplifying desirable characteristics, such as sensitivity and quality factor. The Helmholtz resonance effect arises in geometries containing a narrow neck leading to a larger volume, such as a glass bottle. Due to the relative compliance of the air in the volume and the incompressibility of the air in the neck, a resonance effect is exhibited where the air volume in the cavity is analogous to a mechanical spring, while the air in the neck of the resonator is analogous to a mass.

To the authors' best knowledge, it is the first demonstration of a fully microfabricated acoustic transducer utilizing the Helmholtz resonance effect for sensitivity enhancement. The addition of an acoustic cavity can be simply and inexpensively implemented to greatly improve the performance of similar acoustic transducers. Packaging is also simplified, since resonances within the packaging become less significant.

\section{DESIGN}

The device presented in this paper features a silicon cantilever, with sputter-deposited zinc oxide for piezoelectric sensing, coupled to a microfabricated air cavity. The coupled airacoustic microsystem exhibits acoustic resonance, effectively amplifying the pressure wave which is formed across the cantilever. A schematic representation of the device is shown in Figure 1.

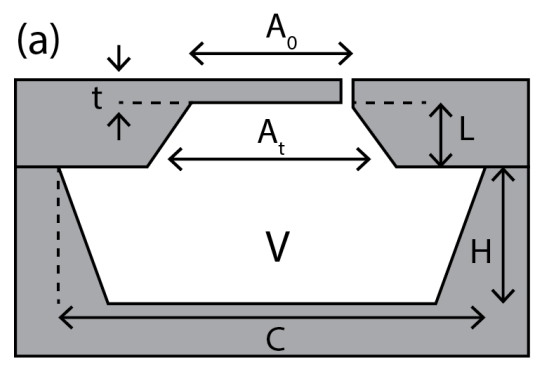

(b)

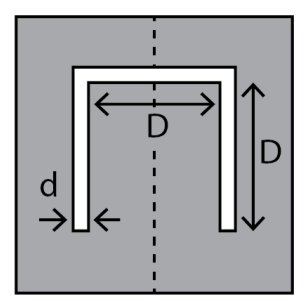

Figure 1: The device schematic with design parameters (a) crosssection view, and (b) top-down view.

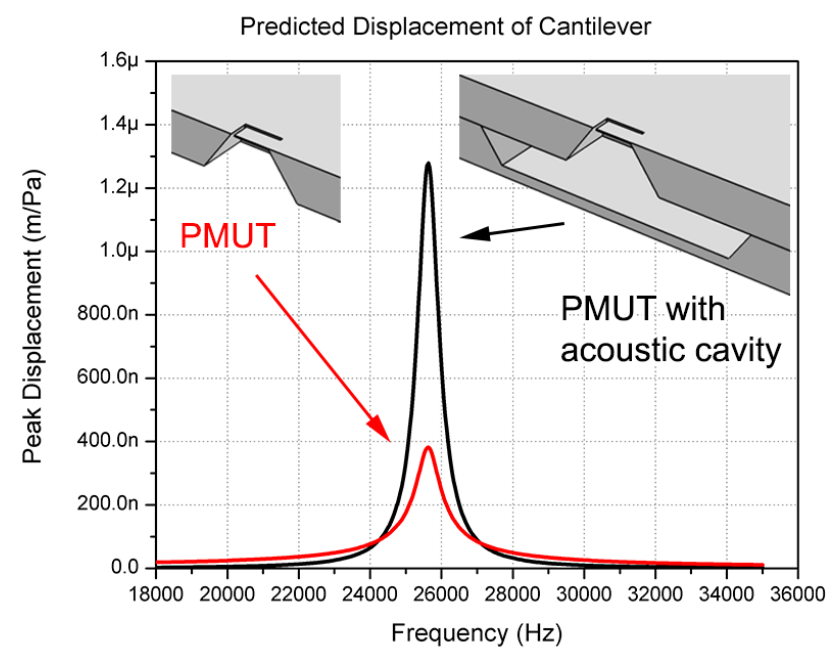

Figure 2: Simulated prediction of the acoustic amplification effect, with pressure amplification factor of 3.35 .

A parametric model was developed to accurately predict the performance and resonance of such a device. This electroacoustic model, first presented in [2], gives an acoustic transfer function of

$$
H(\omega)=\frac{\Delta P(\omega)}{P_{\text {in }}(\omega)}=\frac{R_{G}+j \omega L_{G}}{R_{G}+j \omega\left(L_{G}+L_{N}\right)+1 / j \omega C_{V}}
$$

Where the electroacoustic parameters are related to the geometric parameters by,

$$
\begin{aligned}
C_{V} & =\frac{V}{\rho c^{2}}, & L_{N} & =\frac{\rho(L+\Delta L)}{A_{0} A_{t}} \\
R_{G} & =\frac{12 \eta t}{d^{3}(3 D+2 d)}, & L_{G} & =\frac{6 \eta t}{5 d(3 D+2 d)}
\end{aligned}
$$

A multiphysics finite element model (FEM) based on the COMSOL acoustics module was developed for this geometry. An optimized design was derived for the targeted $26 \mathrm{kHz}$ resonance frequency of the air-acoustic system, with the selected dimensions shown in Table 1.

The analytical model and simulation results closely agree in their prediction of resonance frequency, giving a value of 25.8 $\mathrm{kHz}$. The FEM simulation predicts that a sensitivity improvement by a factor of 3.35 can be achieved with the addition of an acoustic cavity (Fig. 2).

Although the analytical model considers volume of the cavity independently from the actual geometry, simulation results indicated that a shallower volume produces a larger $\mathrm{Q}$ resonance whose center frequency aligns more accurately with analytical predictions. Figure 3 shows that it is easy to tune the acoustic resonance frequency by adjusting the dimension of the air cavity. In this way we can create arrays of acoustic transducers with varying resonance frequencies. This enables summation of the outputs for increased overall bandwidth or measurement from individual elements for use as a bank of notch filters.

Table 1: Summary of the selected geometric design parameters.

\begin{tabular}{|l|c|c|c|c|c|c|c|}
\hline Parameter & $\mathbf{A}_{\mathbf{0}}$ & $\mathbf{t}$ & $\mathbf{L}$ & $\mathbf{D}$ & $\mathbf{d}$ & $\mathbf{C}$ & $\mathbf{H}$ \\
\hline Value $(\mathrm{mm})$ & 0.363 & 0.002 & 0.4 & 0.363 & 0.02 & 2.7 & 0.3 \\
\hline
\end{tabular}

\section{Solid-State Sensors, Actuators and Microsystems Workshop} Hilton Head Island, South Carolina, June 5-9, 2016 


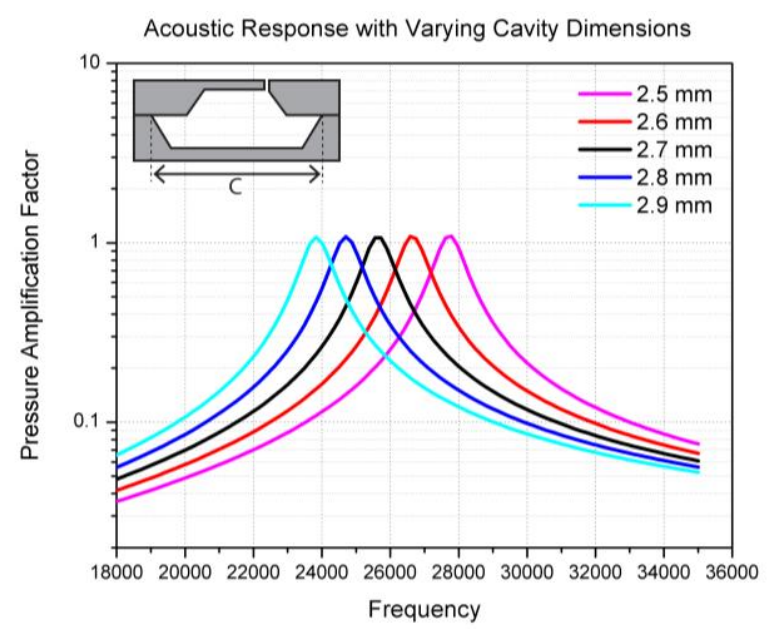

Figure 3: Acoustic frequency response of the device with varying square cavity dimensions.

\section{FABRICATION}

The Helmholtz PMUT is fabricated using a two-wafer approach. An SOI wafer with $2 \mu \mathrm{m}$ thick device layer (Fig. 4i) is used for fabrication of the cantilever-based piezoelectric sensing element. The SOI's buried oxide layer serves as an etch-stop for $\mathrm{KOH}$ backside etching (Fig. 4ii). The sensing layers consist of evaporated $\mathrm{Al}$ electrodes, sputter-deposited $\mathrm{ZnO}$, and PECVD SiN for electrical isolation. DRIE is used for the final release of the paddles (Fig. 4iii).

The Helmholtz resonance chamber is fabricated on a second wafer (Fig. 4iv). Silicon nitride serves as an etch mask for $\mathrm{KOH}$ anisotropic wet etching of silicon. Upon removing the nitride masking layer, bonding is performed using adhesive SU-8 bonding as described in [3]. It is worth noting that a number of bonding methods can be employed, including eutectic bonding, anodic bonding, thermocompressive bonding or glass frit bonding. The completed wafers are diced, wire-bonded, and packaged (Fig. 5).

\section{Cantilever}

(i)

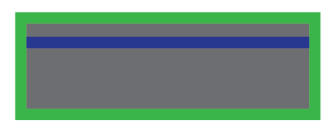

(ii)

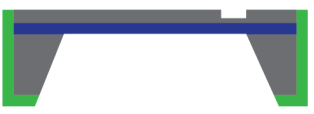

(iii)

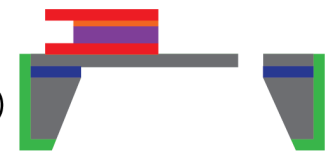

\section{Acoustic Resonator}

(iv)

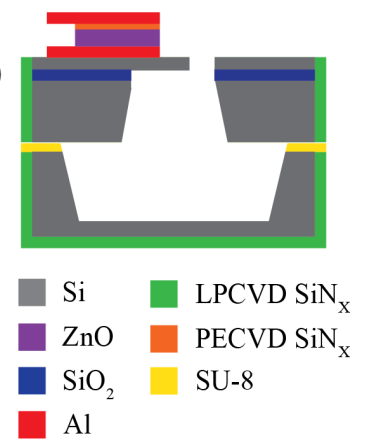

Figure 4: Description of two-wafer fabrication process, consisting of (i) conformal nitride deposition (ii) $\mathrm{KOH}$ etching, (iii) sensing element deposition and patterning, (iv) cavity etching and bonding.

\section{RESULTS AND DISCUSSION}

Device measurements were performed in an anechoic chamber with a GRAS 40AO calibrated microphone serving as a pressure reference. The PMUT output is connected with an OpAmp based preamplifier circuit and enclosed in an aluminum box for electromagnetic shielding.
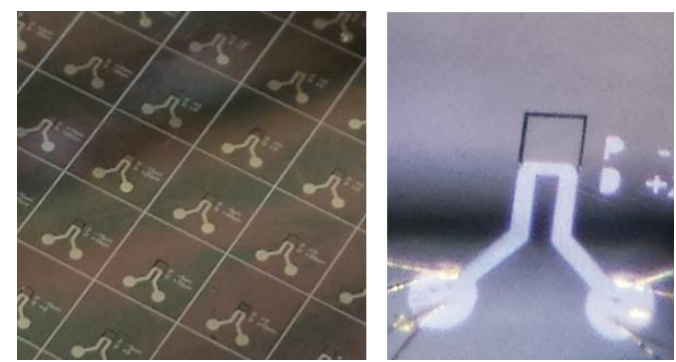

Figure 5: Completed device array (left) and bonded device (right).

The sensitivity of the fabricated PMUT was measured both before and after bonding of the Helmholtz air cavity (Fig. 6). The unamplified sensitivity of the PMUT alone is measured to be 0.297 $\mathrm{mV} / \mathrm{Pa}$ (at $29 \mathrm{kHz}$ with Q of 34.9) and the sensitivity after bonding of the second wafer was $0.64 \mathrm{mV} / \mathrm{Pa}$ (at $29 \mathrm{kHz}$ with Q of 73.0). This corresponds to an improvement in sensitivity by a factor of 2.16 with the use of a matched resonant air cavity.

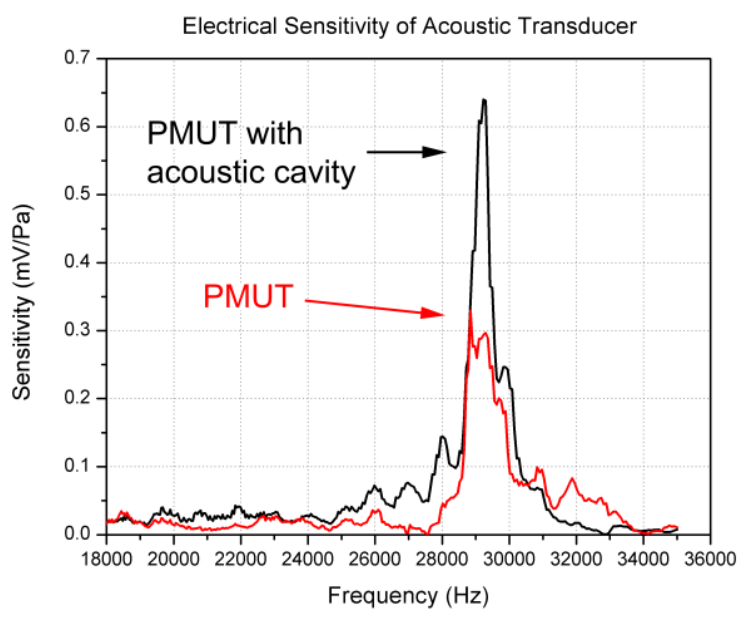

Figure 6: Measured device sensitivity demonstrating amplified sensitivity by a factor of 2.16 .

The presented PMUT can be improved further with additional studies into parameter optimization, as preliminary measurements indicated the potential for sensitivity enhancement of up to two orders of magnitude [2].

\section{CONCLUSION}

These results support the use of Helmholtz resonance as a mechanism for improving sensitivity of microfabricated acoustic sensors. The amplified sensitivity is achieved with minimal additional cost and processing steps, and can be immediately adopted in a variety of existing fabrication flows of acoustic transducers.

\section{REFERENCES}

[1] A.A. Shkel, L. Baumgartel, and E.S. Kim. "A resonant piezoelectric microphone array for detection of acoustic signatures in noisy environments." IEEE International Conference on Micro Electro Mechanical Systems (MEMS), (2015).

[2] A.A. Shkel, E.S. Kim. "Acoustic micro-resonator utilizing hemispherical air cavity for sensitivity enhancement." IEEE International Ultrasonics Symposium (IUS), (2015).

[3] L. Yu, et al. "Adhesive bonding with SU-8 at wafer level for microfluidic devices." Journal of Physics: Conference Series. Vol. 34. No. 1. IOP Publishing, (2006). 